\title{
Association between population migration and epidemic control of coronavirus disease 2019
}

\author{
Yu Ding ${ }^{1,2 \dagger}$, Sihui Luo ${ }^{1,2 \dagger}$, Xueying Zheng ${ }^{1,2 \dagger}$, Ping Ling ${ }^{1}$, Tong Yue ${ }^{1}$, Zhirong Liu $^{3,4}$ \& \\ Jianping Weng ${ }^{1,2,5^{*}}$ \\ ${ }^{1}$ The First Affiliated Hospital of USTC, Division of Life Sciences and Medicine, University of Science and Technology of China, Hefei 230001, \\ China; \\ ${ }^{2}$ Institute of Public Health, University of Science and Technology of China, Hefei 230026, China; \\ ${ }^{3}$ Joint Laboratory of Public Health, USTC and Anhui NHC, Hefei 230001, China; \\ ${ }^{4}$ Anhui Provincial Center for Disease Control and Prevention, Hefei 230601, China; \\ ${ }^{5}$ Division of Life Sciences and Medicine, University of Science and Technology of China, Hefei 230026, China
}

Received February 20, 2020; accepted April 10, 2020; published online April 14, 2020

Citation: Ding, Y., Luo, S., Zheng, X., Ling, P., Yue, T., Liu, Z., and Weng, J. (2020). Association between population migration and epidemic control of coronavirus disease 2019. Sci China Life Sci 63, 936-939. https://doi.org/10.1007/s11427-020-1695-5

Dear Editor,

In January 2020, a widespread outbreak of coronavirus disease 2019 (COVID-19) occurred after the beginning of the largest annual migration in China, which is known as the Spring Festival migration. Starting in January 20, 2020, the Chinese government took a series of unprecedented measures to contain the spread of COVID-19. Because of the role of Wuhan as a central transportation hub, public transportation was suspended in and out of Wuhan and adjacent areas on January 21. Other provinces successively took precautionary actions in accordance with the Level-1 Response to Major Public Health Emergencies (Xinhua News Online, http://www.xinhuanet.com/politics/2020-01/25/ c_1125502232.htm). Nevertheless, the flow of migration prior to these measures had laid the foundation for the accelerated spread of COVID-19. However, the association between the migration patterns of the population and the epidemic control of COVID-19 has not been investigated fully. In this study, we aim to analyze the impact of migration on the epidemic and control of COVID-19 using Hefei and

$\dagger$ Contributed equally to this work

*Corresponding author (email: wengjp@ustc.edu.cn)
Shenzhen as examples.

We chose Hefei and Shenzhen as locales because Hefei is the capital city of Anhui province, which is adjacent to Hubei, where the population inflow during the Spring Festival migration was one of the highest in China; and Shenzhen experienced one of the highest population outflows over the same period. Since the outbreak of COVID19, Hefei (January 24) and Shenzhen (January 23) were the first two regions to launch the Level-1 Response. We defined the period between the launch of the Level-1 Response and 24:00 January 30 as the initial stage of response.

We used an online platform, Baidu Migration ${ }^{\circledR}$ in Baidu Maps $^{\circledR}$, which is one of the largest map service providers in China, as a data source of population migration. We calculated the daily proportion of inflow of population from Wuhan/Hubei to the two cities by dividing the daily number of people moving from the epidemic focus to Hefei or Shenzhen by the total number of daily inflow of people to the two cities, respectively.

The basic reproduction number (R0) reflects the speed of infectious disease spread. We calculated the daily R0 based on serial, overlapping, 5-day time windows for Hefei and Shenzhen, respectively, over the study period between January 19 and February 11, 2020. We adopted a 5-day window 
because the previously reported incubation period of COVID-19 was approximately 5 days ( $\mathrm{Li}$ et al., 2020). R0 was estimated using the basic method of $\mathrm{R} 0 \geq(1+$ growth rate $\times$ serial interval).

We estimated the serial interval by adding the average incubation period (days) to the average infectious period (days). The average growth rate, average incubation period, and average infectious period were calculated at the end of each time window by simple moving averages for the two cities, respectively.

To obtain the profile of confirmed cases of COVID-19 in Hefei and Shenzhen, we extracted the data of confirmed cases from the websites of the municipal Centers for Disease Control and Prevention (CDC) of the two cities. The first confirmed case occurred on January 19 in Shenzhen, which was defined here as the starting point of the outbreak.

The basic characteristics of the confirmed cases in the two cities are shown in Table S1 in Supporting Information. By 24:00 on February 11, 157 confirmed cases had been identified in Hefei, aged $44.40 \pm 15.60$ years, 74 female $(47.1 \%)$ and 38 imported cases (24.2\%). During the same period, 386 confirmed cases had been identified in Shenzhen, aged $45.15 \pm 17.99$ years, 202 female $(52.3 \%)$ and 289 imported cases $(74.9 \%)$. Hefei had a significantly lower proportion of imported cases compared with Shenzhen $(P<0.001)$. The number of daily newly confirmed cases in Hefei exhibited a modest fluctuation. In contrast, in Shenzhen, the increase in the number of cases was slow during the week that followed the identification of the first case, but accelerated immediately after, reaching a peak of 60 new cases on January 31. Thereafter, the number of new cases decreased quickly (Figure S1 in Supporting Information). To understand the changes in the epidemiological characteristics of COVID-19 (Figure 1), we evaluated the change in R0 over the study period in Hefei and Shenzhen based on a slope prediction algorithm. The first order functions of time and R0 were obtained using the method of least squares. As shown in Figure $1 \mathrm{~A}$, the daily R0 values were $>3.5$ in both cities before the Level-1 Response. After the launch of the Level-1 Response, the R0 values in both cities started to decline slowly (both, $P$ for trend $<0.001$ ). By February 11 , the R0 in Hefei and Shenzhen were 1.93 and 1.48 , respectively. Moreover, compared with Hefei, the decline in R0 in Shenzhen was apparently faster (predicted slope of R0, Shenzhen vs. Hefei, -0.133 vs. $-0.031, P=0.091$ ). Regarding the components used in the estimation of R0 shown in Figure $1 \mathrm{~B}$, the average growth rate declined significantly in both cities after the initial stage of response (both, $P$ for trend $<0.001)$. The average incubation period of both the cities increased over time (both, $P$ for trend $<0.001$, Figure $1 C$ ). Figure 1D indicates that the average infectious period in both cities dropped after the launch of the Level-1 Response, but slowly increased after the initial stage of the response (both,
$P$ for trend $<0.05$ ).

We analyzed the association between $\mathrm{R} 0$ and the population inflow from the epidemic focus in both cities using Spearman's correlation. During the initial stage of the response, no significant association was observed between R0 and the population inflow from the epidemic focus $(P=0.260$, $r=-0.452)$ in Shenzhen; in contrast, in Hefei, the association between $\mathrm{R} 0$ and the population inflow from the epidemic focus was strong $(P=0.000, r=1.0)$. However, after the initial stage of the response, the situation was reversed. A weak association was observed between the $\mathrm{R} 0$ and the proportion of population inflow from the epidemic focus in Shenzhen $(P=0.073, r=0.536)$, but not in Hefei $(P=0.498, r=0.217)$ (Table S2 in Supporting Information).

In this study, we found that, after the launch of the Level-1 Response, the spread rate of COVID-19 declined significantly in both cities analyzed. The consistent decline in R0 recorded during our study period in both cities could be attributed to the consistent decline in the average growth rate, as the average incubation period and average infectious period increased consistently. We assume that such decline in $\mathrm{R} 0$ could be attributed to the multiple strategies employed in the Level-1 Response in the two cities, including raising public awareness, extensive surveillance, and strengthened quarantine strategies, which are proven effective epidemic control measures (Munster et al., 2020).

We found that there were different patterns in the epidemic of COVID-19 in the two cities: the major proportion of confirmed cases in Shenzhen during the study period were imported cases, while Hefei exhibited predominantly clustered cases. The explanation for this phenomenon may be multifactorial; however, population migration and social connections may have played an important role (Merler and Ajelli, 2010).

In Hefei, before the Level-1 Response, a major inflow of population from Hubei/Wuhan was observed, which brought in imported cases. Therefore, during the initial stage of the response, the R0 was strongly correlated with this population inflow. However, this inflowing population was composed mainly of Anhui natives who were returning home. They tended to have extensive local social connections and attended gatherings with the local people frequently. These gatherings accelerated the local COVID-19 spread and generated secondary cases. These local cases further spread the disease, even after the travel ban of Wuhan. This would explain the prevalence of clustered cases and the diminishing of the correlation between R0 and the population inflow observed after the initial stage of the response.

In Shenzhen, the initial population flow consisted of individuals that were moving out of the city. Therefore, during the first few days, no significant correlation with the migration flow was observed. However, during the $24 \mathrm{~h}$ that followed the announcement of the travel ban of Wuhan, a 

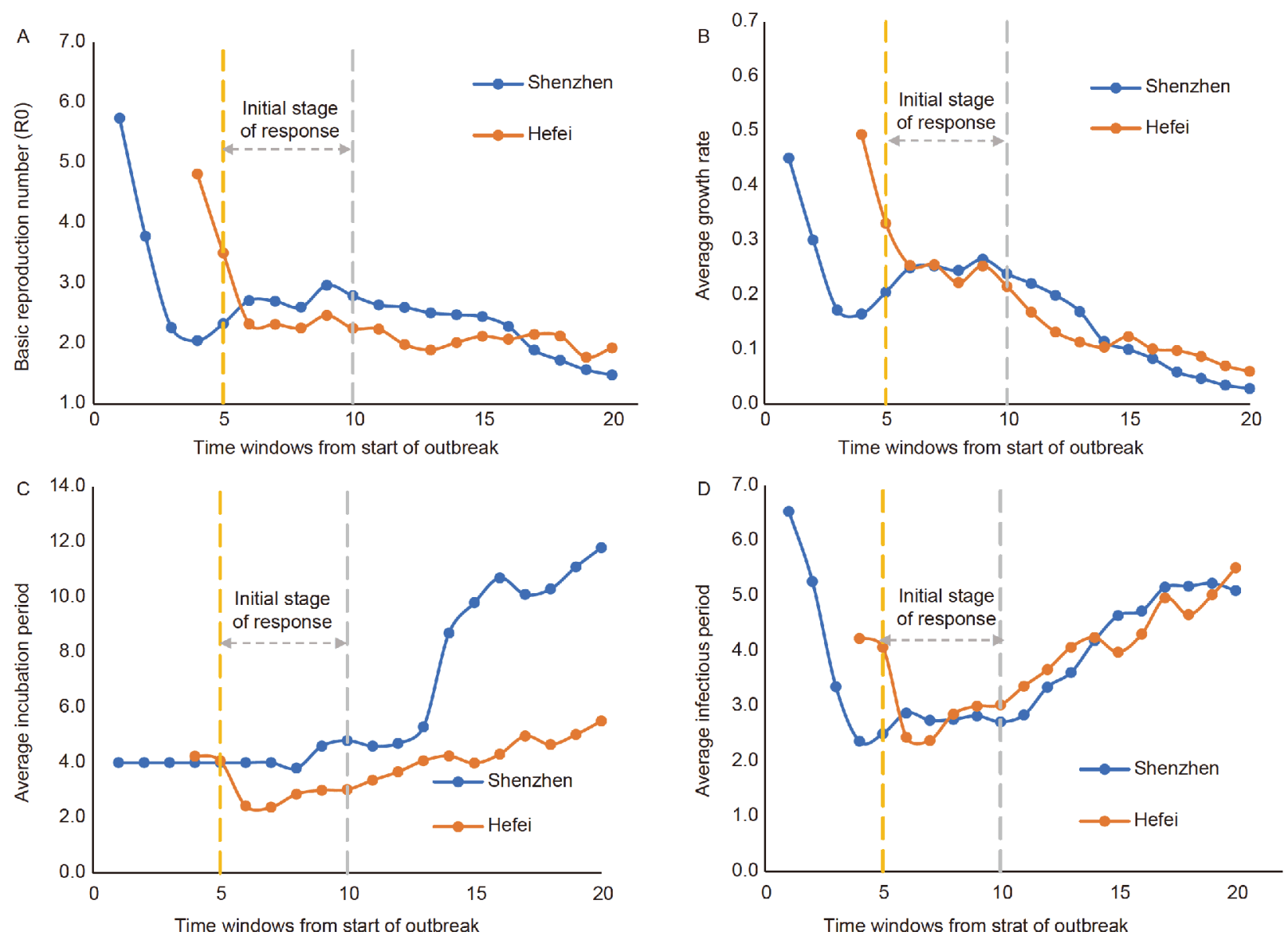

Figure 1 Changes in the epidemiological characteristics of COVID-19 infections recorded in Shenzhen and Hefei between January 19 and February 11 , 2020. A-D, Changes in the basic reproduction number (R0), the average growth rate, the average incubation period (days), and the average infectious period (days) recorded between January 19 and February 11, 2020, in Shenzhen and Hefei. This period was divided into 20 overlapping 5-day time windows. The growth rate referred to the proportion of newly added patients per day in the cumulative total of confirmed patients. The simple moving average of growth rates for the previous 5 days was defined as the average growth rate within a time window ending on a certain date. The average incubation period was the mean value of the incubation period of non-input confirmed patients with a contact history over the previous 5 days. We defined the infectious period as the period between the appearance of the first symptoms and isolation (hospitalization). Similarly, we defined the average infection period as the moving average of all confirmed COVID-19 cases for each 5-day time window.

sudden large inflow from Wuhan was observed that was composed of fleeing populations. This would explain the burst of new cases recorded on January 31, despite the launch of the Level-1 Response, which exhibited a 7-day time lag that was consistent with the reported incubation period (Li et al., 2020). These new cases were less likely to attend gatherings and were quickly identified after the implementation of disease-control actions. This would explain the large proportion of imported cases observed in Shenzhen.

Overall, population migration and social connection seemed to affect the pattern of spread of COVID-19. This indicated that, to control the spread of COVID-19, differentiated strategies are required that consider both population flow and cultural factors. Under a novel infectious disease outbreak background, the trend of population migration should be closely monitored in advance, and a quick response should be implemented to deal with expected and unexpected population flow events. For cities like Hefei, which has a predominant inflow of native populations from the epidemic focus, the restriction of gatherings would be a priority. For cities like Shenzhen, close monitoring and a quick response to an unexpected large inflow or outflow of population would help prepare an adequate surveillance strategy and allocate healthcare resources.

This was the first report of the association between population migration patterns and the epidemic characteristics and disease control of COVID-19. These results have major implications for public health, in that resources should be allocated according to population flow and its cultural impact. The limitations of this study included the fact that we were only able to access aggregated data, which precluded a more accurate estimation of the R0.

In conclusion, after the implementation of the Level-1 Response, a consistent decline in the R0 of COVID-19 was observed in both Hefei and Shenzhen. The two cities exhibited different patterns of disease spread, which were dri- 
ven by different patterns of population migration. This indicated that population migration should be considered when setting policies aimed at controlling a novel infectious disease.

Compliance and ethics The author(s) declare that they have no conflict of interest.

Acknowledgements This study was supported by the Fundamental Research Funds for the Central Universities (YD9110004001 to JW, YD9110002002 to XY) and Emergency Research Project of Novel Coronavirus Infection of Anhui Province (202004a07020002 to ZRL;
$202004 a 07020004$ to ZRL).

\section{References}

Li, Q., Guan, X., Wu, P., Wang, X., Zhou, L., Tong, Y., Ren, R., Leung, K. S.M., Lau, E.H.Y., Wong, J.Y., et al. (2020). Early transmission dynamics in Wuhan, China, of novel coronavirus-infected pneumonia. N Engl J Med 382, 1199-1207.

Merler, S., and Ajelli, M. (2010). The role of population heterogeneity and human mobility in the spread of pandemic influenza. Proc R Soc B 277, $557-565$.

Munster, V.J., Koopmans, M., van Doremalen, N., van Riel, D., and de Wit, E. (2020). A novel coronavirus emerging in China-key questions for impact assessment. N Engl J Med 382, 692-694.

\section{SUPPORTING INFORMATION}

Figure S1 Daily newly confirmed cases of COVID-19 in Hefei and Shenzhen during the study period.

Table S1 Basic characteristics of the confirmed cases of COVID-19 in Hefei and Shenzhen

Table S2 Basic reproduction number (R0) and population inflow from the epidemic focus recorded in Shenzhen and Hefei between January 23 and February 11, 2020

The supporting information is available online at http://life.scichina.com and https://link.springer.com. The supporting materials are published as submitted, without typesetting or editing. The responsibility for scientific accuracy and content remains entirely with the authors. 